\title{
An Account of Natural Language Coordination in Type Theory with Coercive Subtyping ${ }^{\star}$
}

\author{
Stergios Chatzikyriakidis ${ }^{1}$ and Zhaohui $\mathrm{Luo}^{2}$ \\ 1 Dept of Computer Science, Royal Holloway, Univ of London \\ Egham, Surrey TW20 0EX, U.K; Open University of Cyprus \\ stergios.chatzikyriakidis@cs.rhul.ac.uk, \\ 2 Dept of Computer Science, Royal Holloway, Univ of London \\ Egham, Surrey TW20 0EX, U.K; Open University of Cyprus \\ zhaohui@cs.rhul.ac.uk
}

\begin{abstract}
We discuss the semantics of NL coordination in modern type theories (MTTs) with coercive subtyping. The issue of conjoinable types is handled by means of a type universe of linguistic types. We discuss quantifier coordination, arguing that they should be allowed in principle and that the semantic infelicity of some cases of quantifier coordination is due to the incompatible semantics of the relevant quantifiers. NonBoolean collective readings of conjunction are also discussed and, in particular, treated as involving the vectors of type $\operatorname{Vec}(A, n)$, an inductive family of types in an MTT. Lastly, the interaction between coordination and copredication is briefly discussed, showing that the proposed account of coordination and that of copredication by means of dot-types combine consistently as expected.
\end{abstract}

\section{Introduction}

The literature on NL coordination dates back to [22] and a number of proposals have been put forth within the Montagovian tradition since then. However, a number of central issues as regards NL coordination have not been clarified yet. In this paper we depart from single-sorted versions of type theory found in Montague's work (as well as in most of the subsequent work within the same tradition) and employ a many-sorted modern type theory (MTT) ${ }^{3}$, as proposed and studied for NL semantics in $[30,17,18]$, to deal with two central issues in NL coordination. These issues concern the notion of conjoinable types, in effect the question of which NL elements can be coordinated, and non-Boolean conjunction, where a collective rather than the expected Boolean distributive reading of and arises. The difference between collective and distributive readings is exemplified

\footnotetext{
* This work is supported by the research grant F/07-537/AJ of the Leverhulme Trust in the U.K.

${ }^{3}$ Examples of modern type theories include Martin-Löf's type theory [21,26], the Unifying Theory of dependent Types (UTT) [15] and the type theory implemented in the Coq proof assistant (pCIC) [7].
} 
in the examples below, where the same conjoined NP is interpreted distributively in (1) but collectively in (2):

(1) John and Mary came to the Party.

(2) John and Mary met at the Party.

We shall investigate how collective readings can be interpreted by means of the inductive family of types of vectors in an MTT.

We further discuss the interaction between dot-types for coordinated NPs. Dot-types have been proposed by Pustejovsky [28,29] for lexical interpretations of inherently polysemous words in phenomena such as co-predication (see, for example, [2]). ${ }^{4}$ For example, book according to [28] can be represented with the dot-type PHY - INFO, a type whose objects have both a physical and an informational aspect. Dot-types have been formally introduced into MTTs with coercive subtyping $[17,18]$ and a computational implementation of this account in Plastic ${ }^{5}$ has also been done [35]. What we want to look at in this paper is the interaction between these types and coordination, i.e. examples of the following sort:

(3) The book and my lunch were sent by mistake to someone else.

(4) John picked up the newspaper and the book from the floor.

Given that the dot-types of the coordinated phrases are different and assuming that the NL coordination operate on the same types, we will have to explain how coordination is possible in these cases. The problem that arises in examples like (3) and (4) is that the individual NPs of the conjunction (e.g. the book and my lunch in (3) have different types (PHY $\bullet$ INFO for book and EvEnT $\bullet$ PHY for lunch). The challenge is to account for the possibility of coordination in these cases by, at the same time, retaining the assumption that coordination operates on elements of the same type. As we shall see, the coercive subtyping mechanism actually allows us to combine the proposed typing for NL coordinations and the account with dot-types in a rather straightforward way.

\section{Type Theory with Coercive Subtyping}

In this paper, we employ modern type theories (MTTs) as the language for formal semantics. A brief introduction to the relevant features of MTTs are briefly given here.

An MTT has a number of differences when compared to Church's simple type theory as employed in Montague semantics [6,23]. One of the most important

\footnotetext{
${ }^{4}$ See also [3] for a critique of the flaws in the various formalizations of dot-types in their original formulation as well as in much of the later work based on that.

${ }^{5}$ Plastic [5] is a proof assistant, an implementation of the modern types theory UTT [15] on the computer for formalised proof development. In the context of linguistic semantics, type theory based proof assistants such as Agda [1], Coq [7] and Plastic can be used to formalise and reason about the formal semantics based on MTTs.
} 
differences between an MTT and the simple type theory, is that the former can be regarded as many-sorted while the latter single-sorted. MTTs use many types to interpret Common Nouns $(\mathrm{CN})$ such as man and table, while single-sorted type theories use only one type $(e)$ for the type of all entities (and another type $t$ for logical truth values), with CNs being interpreted as predicates of type $e \rightarrow t$.

In Montague semantics, an Intransitive Verb (IV) is interpreted as a function from entities to truth values $(e \rightarrow t)$, a type which is shared with CNs and intersective adjectives, and a quantified NP as of the type from properties to truth values $((e \rightarrow t) \rightarrow t)$.

In an MTT, types ('sorts') are used to interpret the domains to be represented. Some of them are:

- the propositional types (or logical propositions),

- the inductive types such as the type of natural numbers and $\Sigma$-types of dependent pairs,

- the inductive families of types such as the types $\operatorname{Vec}(A, n)$ of vectors (or lists) of length $n$, and

- other more advanced type constructions such as type universes.

For example, within such a many-sorted logical system CNs are not interpreted as predicates as in the Montagovian tradition but rather as Types. Theoretical motivation behind such a proposal has been provided by the second author based on the notion of identity criteria that CNs have according to [9]. (See [19] for the exact details of this proposal.) Then given the interpretation of CNs as types, adjectives are interpreted as a predicate over the type interpreting the domain of the adjective. For example, the adjective handsome is interpreted

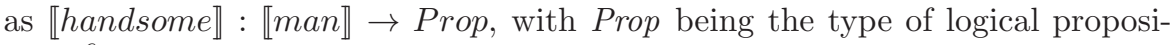
tions. ${ }^{6}$ Modified CNs are then interpreted as $\Sigma$-types, the types that intuitively represent subset types but contain explicit proof objects. ${ }^{7}$

One of the important features of MTTs is the use of dependent types. Two examples of basic constructors for dependent types are $\Pi$ and $\Sigma$. The $\Pi$-type corresponds to universal quantification in the dependent case and implication in the non-dependent case. In more detail, when $A$ is a type and $P$ is a predicate over $A, \Pi x: A . P(x)$ is the dependent function type that, in the embedded logic, stands for the universally quantified proposition $\forall x$ :A.P $(x)$. A $\Pi$-type degenerates to the function type $A \rightarrow B$ in the non-dependent case. In the case of $\Sigma$, if $A$ is a type and $B$ is an $A$-indexed family of types, then $\Sigma(A, B)$, or sometimes written as $\Sigma x: A . B(x)$, is a type, consisting of pairs $(a, b)$ such that $a$ is of type

\footnotetext{
${ }^{6}$ MTTs have consistent internal logics based on the propositions-as-types principle $[8,14]$. For example, in a predicative type theory such as Martin-Löf's type theory, the logical proposition $A \& B$ corresponds to the product type $A \times B$ (a special case of $\Sigma$-type - see below) and a pair of a proof of $A$ and a proof of $B$ corresponds to an object of the product type. In an impredicative types theory such as UTT, logical propositions are similarly constructed as types but, furthermore, there is the type Prop - a totality of logical propositions.

${ }^{7}$ See $[30,17]$ for more details on this.
} 
$A$ and $b$ is of type $B(a)$. When $B(x)$ is a constant type (i.e., always the same type no matter what $x$ is), the $\Sigma$-type degenerates into product type $A \times B$ of non-dependent pairs. $\Sigma$-types (and product types) are associated projection operations $\pi_{1}$ and $\pi_{2}$ so that $\pi_{1}(a, b)=a$ and $\pi_{2}(a, b)=b$, for every $(a, b)$ of type $\Sigma(A, B)$ or $A \times B$.

Coercive subtyping is an adequate subtyping mechanism for MTTs $[16,20]$ and, in particular, it avoids a problem associated with the ordinary notion of subtyping (subsumptive subtyping), namely violation of canonicity [17]. ${ }^{8}$ Basically, coercive subtyping is an an abbreviation mechanism: $A$ is a (proper) subtype of $B(A<B)$ if there is a unique implicit coercion $c$ from type $A$ to type $B$ and, if so, an object $a$ of type $A$ can be used in any context $\mathfrak{C}_{B}[-]$ that expects an object of type $B: \mathfrak{C}_{B}[a]$ is legal (well-typed) and equal to $\mathfrak{C}_{B}[c(a)]$. For instance, one

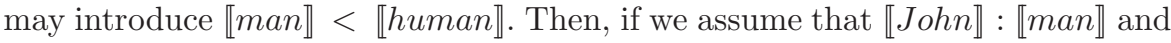

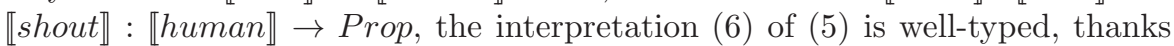

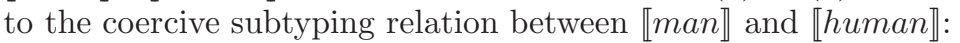

(5) John shouts.

(6) $\llbracket$ shout $\rrbracket(\llbracket J o h n \rrbracket)$

Ending our discussion on the preliminaries of TTCS, we mention one further more advanced feature of the theory, that of universes. A universe is a collection of (the names of) types into a type [21]. This can be seen as a reflection principle where the universe basically reflects the types whose names are its objects. Universes are extremely useful in accounts of lexical semantics using MTTs. Specifically, universes can help semantic representations. To give an example, one may use the universe CN : Type of all common noun interpretations and, for each type $A$ that interprets a common noun, there is a name $\bar{A}$ in $\mathrm{CN}$. For example,

$$
\overline{\llbracket m a n \rrbracket}: \mathrm{CN} \quad \text { and } \quad T_{\mathrm{CN}}(\overline{\llbracket m a n \rrbracket})=\llbracket \operatorname{man} \rrbracket .
$$

In practice, we do not distinguish a type in $\mathrm{CN}$ and its name by omitting the overlines and the operator $T_{\mathrm{CN}}$ by simply writing, for instance, man $\rrbracket$.

Summarizing, we can say that the use of TTCS in interpreting NL semantics has given a number of interesting results and insights. These include an increased type granularity when compared to Montague Semantics given its type richness as well as an adequate subtyping mechanism. ${ }^{9}$ Furthermore the interpretation of CNs as Types rather than predicates seems to be closer to the idea according to which the distinguishing feature of CNs, when compared to other parts of speech, is that only the former have what Geach called, criteria of identity [9]. The work presented in [19] provides strong arguments for supporting the non-predicate view on CNs based on Geach's identity criteria. The successful formalization [17] and subsequent implementation in Plastic [35] of dot.types is another achievement of this line of research given that no proper formalization

\footnotetext{
8 See [17] for the notion of canonicity.

${ }^{9}$ This subtyping mechanism is however in line with canonicity and as such computationally more attractive [17].
} 
of dot.types existed up to that point. The use of universes has been also proven fruitful in looking at alternative ways for defining the types for quantifiers and adverbs among others. Lastly, parts of the various proposals made in the aforementioned papers have been tested using the Coq interactive theorem prover. Some first results can be seen in [18] as well as in this paper. Current work of the first author concentrates on the use of Coq to prove valid NL theorems ${ }^{10}$ as well as building universes relevant to NL semantics (e.g. CN, LType) in Plastic. ${ }^{11}$

\section{Conjoinable Types}

The issue of defining which NL types are conjoinable is of very high importance to all accounts of coordination proposed so far. Most of the accounts that have been proposed in the Montagovian tradition argue that conjoinable types are either of type $t$ or of a function type that ends with $t$. The formalization might be different in individual cases but the core of the proposal is pretty much the same. The definition as given by Winter [34] is given below (using the term t-reducible): ${ }^{12}$

(7) $\tau$ is a t-reducible type iff $\tau=t$ or $\tau=\tau_{1} \tau_{2}$, where $\tau_{1}$ is any type and $\tau_{2}$ is a t-reducible type.

Such type of formulation allows coordination of categories ending in type $t$ only, with type $e$ conjunction not being possible. Thus, in these accounts proper name coordination is either assumed to involve type-lifting to quantifier type or proper names are assumed to be quantifiers in all cases. However, Partee \& Rooth [27] propose a definition of $e$ conjoinable types to deal with collective reading cases. Similar proposals have been made by Hoeksema [11]. Of course, an inductive definition of an e-conjoinable type does not make much sense given that at least in standard Montagovian semantics, the only $e$ conjoinable types are the type of individual concepts, of type $s \rightarrow e$, i.e the type from indices to individuals, so the definition basically covers just one case.

Moving away from the simple type theory in Montague Grammar and using many-sorted MTTs, the first question to ask ourselves is how conjoinable categories can be defined. Well, the first question to be asked is which linguistic types can be conjoined? Surprisingly (or not) it seems that all linguistic categories can be conjoined. We first note the obvious cases of sentence and predicate coordination (8 and 9) to CN coordination (10):

(8) John walks and Mary talks.

(9) John walks and talks.

\footnotetext{
${ }^{10}$ An example of this type is the following: if John and Mary met then John met Mary. Such theorems can be proved if the correct semantics are given in each case.

11 This is not possible in Coq.

${ }^{12}$ We follow the notation as this is given in [34]. As such, $\tau_{1} \tau_{2}$ should be taken to mean $\tau_{1} \rightarrow \tau_{2}$.
} 
(10) A friend and colleague came.

Then, quantified NP coordination (11), quantifier coordination (12) and proper name $(\mathrm{PN})$ coordination are possible (13):

(11) Every student and every professor arrived.

(12) Some but not all students got an A.

(13) John and Mary went to Italy.

Adverb conjunction(14), preposition conjunction(15), PP conjunction (16)

(14) I watered the plant in my bedroom but it still died slowly and agonizingly.

(15) I can do with or without you.

(16) The book is on the table and next to the chair.

Lastly, coordination of subordinate connectives is also possible (17):

(17) When and if he comes, you can ask him.

\subsection{Universe of Linguistic Types}

In this section we will propose a way to handle the flexibility NL coordination exhibits by using a MTT. The key idea behind the account we are going to propose is the notion of a universe.

A universe, as we have already mentioned at the end of $\S 2$, is a collection of (the names of) types into a type [21]. In the case of coordination, the universe CN of the types that we have used to interpret common nouns is far too small to capture the generality of the phenomenon. Given that all linguistic categories can be coordinated, the universe we need, has to be far more general than CN.

The idea is to introduce a type universe LType of Linguistic Types. Intuitively, LType contains (the names of) all types that are employed in linguistic semantics. Of course, in doing so, we will have to specifically say what we consider a linguistic type to be. Even though a thorough discussion of meticulously constructing the universe of linguistic types is out of the scope of this paper, we shall indicate positively what types may have names in the universe LType. ${ }^{13}$ Figure 1 contain some of the introduction rules for LType, where we have used the so-called Russell-style formulation of a universe to omit the names of its objects. The informal explanations of the rules in Figure 1 are given below.

- The type Prop of logical propositions is a linguistic type. (It is of type PType ${ }^{14}$ by the first rule and hence of type LType by the last rule.)

${ }^{13}$ We leave this most thorough and complete discussion of the universe LType for future work.

${ }^{14}$ Ptype can be thought of as the universe of predicates. It is an intermediate universe used to build LType. 


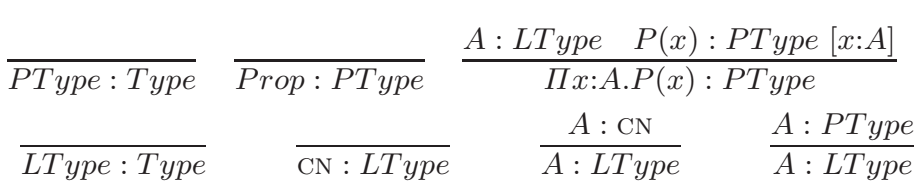

Fig. 1. Some (not all) introduction rules for LType.

- If $A$ are linguistic types and $P$ is an $A$-index family of types in PType, so is the $\Pi$-type $\Pi x: A . P(x)$. In particular, in the non-dependent case, if $A_{i}$ are linguistic types, so is the arrow type $A_{1} \rightarrow \ldots \rightarrow A_{n} \rightarrow$ Prop. (It is of type PType by repeated uses of the third rule and hence of type LType by the last rule.)

- The universe $C N$ (of types that interpret common nouns) is an object of type LType.

- If $A$ interprets a common noun, then $A$ is a linguistic type in LType. For example, the $\Sigma$-types that interpret modified CNs are in LType.

Other example types in LType include the type of VP adverbs and that of quantifiers, shown in the following examples:

(18) $\Pi A:$ CN. $(A \rightarrow$ Prop $) \rightarrow(A \rightarrow$ Prop $)$

(19) $\Pi A:$ CN. $(A \rightarrow$ Prop $) \rightarrow$ Prop

Please note that we have only listed some of the introduction rules for LType. For example we have not yet included the type for PP-modifiers. At the moment, we shall leave the universe LType to be open in the sense that we may introduce new types into it in the future. ${ }^{15}$

Having described the universe of linguistic types, we can now use it to describe the type of coordinators: every (binary) coordinator is of the following type:

(20) $\Pi A:$ LType. $A \rightarrow A \rightarrow A$

For instance, the coordinator and is of the above type.

To give an example of how this type works, let us imagine three cases of coordination: PN coordination (John and George), propositional coordination (John runs and Mary drives) and VP coordination (John cycles and drives). In the first case, John and George are of type $\llbracket M a n \rrbracket$, so the $A$ in this case is of type $\llbracket$ Man》 which is in LType given that it is of type $C N$. In the case of propositional coordination, our $A$ is of type Prop, which being a PType is also

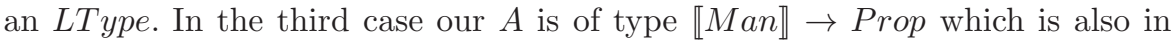
LType. Similar considerations apply to all the cases from (8) to (17). Thus, this type captures the flexibility associated with coordination. ${ }^{16}$ It is not difficult to

${ }^{15}$ Formally, openness of a universe would imply that we do not impose an elimination rule for it. We omit the technical details here.

${ }^{16}$ Of course, there are cases discussed in the literature where coordination of different categories seems to be possible. One such example is discussed in [24], where an 
see that all examples of coordination from (8) to (17) are predicted via the type given for coordination above. ${ }^{17}$ However, what we need to discuss is examples where the rule proposed in (20) might seem to overgenerate or departs from the standard assumptions as these are made in the formal semantic literature.

\subsection{Quantifier Coordination}

The type for coordination we have proposed might be argued to overgenerate for cases involving coordination of two quantifiers like the ones shown below:

(21) \# Some and every man came

(22) \# No and some boy read

The above sentences seem to be generated via the rule we have proposed for coordination. Note, that this problem applies to all coordination accounts proposed. Given that quantifiers involve a function type ending in $t$, they should be conjoinable according to the accounts proposed in the Montagovian literature. No explicit discussion has been made of how cases like these are disallowed, so it would be good to see in more detail what is going on in these cases.

The basic problem is that some quantifiers seem to be able to be coordinated and some others do not. Between the cases of quantifiers that cannot be coordinated with a coordinator there are cases where adding a modal adverb between the coordinator and the second conjunct make a difference in acceptability. For example, adding the modal adverb possibly in (21) but not in (24) makes the sentence semantically felicitous:

(23) Some and possibly every man came

(24) \# No and possibly some boy read

For the rest of the cases, whether such sentences will be semantically felicitous depends on the type of coordination in each case (cf. the two examples below):

(25) \# One and two of my students came to the party.

(26) One or two of my students came to the party.

So, it seems that in principle, we should allow coordination of quantifiers, since there are clear cases where this is possible. However, allowing coordination of quantifiers to be in principle possible, we will have to explain semantically infelicitous cases like (25). A way that can help us rule out a number of infelicitous semantic cases is to look at the semantics of the individual quantifiers in

adjective is coordinated with a NP: John is either stupid or a liar. We will not pursue an account here but we could note that an account in a similar vein to the one proposed by [25] where coordination even in this case operates on like and not on unlike categories is possible.

17 All the examples have been checked using the Coq theorem prover [7]. The code can be found in the Appendix. 
combination with the coordinator in each case. Let us take the example of the following NP:

(27) \# Some and no men arrived.

The quantifiers in the above example can be coordinated via the rule we have proposed. However, the semantics we get for the coordinated NP some and no man are the following, in effect a contradiction:

$$
\text { (28) } \exists x: \llbracket \operatorname{man} \rrbracket . P(x) \wedge \sim \exists x: \llbracket \operatorname{man} \rrbracket . P(x)
$$

We can quite plausibly assume that the contradictory semantics is the reason the conjunction is infelicitous in (31), especially when uttered out of the blue without any context. Now imagine the following situation: someone is lying and has stated that no men arrived on one occasion and that some men arrived on another. Then, the hearer might spot this contradiction and utter the following 'some and no men arrived?'. In this case, some and no men is perfectly felicitous. ${ }^{18}$ Disjunction of the same quantifiers is possible without the aid of some special context. Examples of this quantifier combination are quite frequently found in NL:

(29) People with some or no academic training.

(30) This license may grant the customer the ability to configure some or no parts of the software themselves.

The semantics of some or no $x$ in contrast to the semantics of some and no $x$ do not give rise to a contradiction. To the contrary, they are always true under any interpretation. The example below depicts the semantics of some or no men: ${ }^{19}$

$$
\text { (31) } \exists x: \llbracket \operatorname{man} \rrbracket P(x) \vee \sim \exists x: \llbracket \operatorname{man} \rrbracket . P(x)
$$

Further examples of quantifiers that do not need a special context are some but not all, more than three and less than five. It might then be the case, that quantifier combinations that are always false need a special context in order to be felicitous while quantifier combinations that do not fall into this category do not. Of course, there are obvious counterexamples to such a proposal, for example cases like some and all or most and all, which are of course infelicitous in the absence of any special context contrary to what we expect in case what we say is true. However, quantifiers like some and most in NL carry a quantity implicature (see e.g. [13], [12] and [10] among others). The idea is that a speaker uttering some and not the stronger all, does that because he believes that substitution for the stronger value cannot be done salva veritate. For if the latter was true, he would have uttered the stronger all. A quantifier combination like some and all cancels out this implicature, so this might be the reason for the infelicitousness of

18 The same kind of example can be devised for cases like (23).

19 This is the case assuming a logical interpretation of some. If the quantity implicature is taken into consideration, the quantifier combination is not always true. 
this quantifier combination when uttered out of context. The same can be argued for the case of most and all. The issue requires more careful examination in order to see whether what we have argued is true or not. In particular, one has to check whether cases of quantifier combinations that are always false need the aid of some special context in order to be felicitous. Then, cases where infelicitousness arises unexpectedly must be shown to arise from other independent factors (like the quantity implicature for example). We believe that what we have proposed can produce a fruitful line of research as regards quantifier coordination but at least for this paper, we will not examine the issue any further. What is rather uncontroversial, no matter the assumptions we make as regards the interplay of quantifier coordination and the use of context, is that we need a rule for coordination that will in principle allow quantifier coordination. The rule we have proposed in (24) suffices for this reason.

Recapitulating, we propose a general rule for coordination which extends over a universe that contains all linguistic types, the universe LType. This rule is general enough to allow all types of coordination we find in NL. The rule might seem to overgenerate in the case of quantifier coordination, but as we have seen, in principle quantifier coordination should be allowed. The infelicitous cases (when uttered out of the blue) are attributed to the semantics of the individual quantifiers under the coordinator involved in each case.

\subsection{Non-Boolean Conjunction}

The first thing we have to see is what is the prediction our typing rule proposed for coordination makes for these cases. But before doing this, we first have to discuss the typing of predicates like meet in their collective interpretation. Such predicates can be seen as one place predicates that take a plural argument and return a logical proposition (something in Prop in our case), an assumption already made in a number of the accounts within the Montagovian tradition (e.g. [34,33]). The plausible question is how plural arguments are going to be represented.

An interesting account of plurality within an MTT is presented by [4], where Martin-Löf's type theory is used for linguistic semantics. In this account, plural count nouns are interpreted using the type List $(A)$. Such an account is shown to give a uniform treatment of both singular and plural anaphora, being compatible with the classical type-theoretic treatment of anaphora, as this is given by [32]. ${ }^{20}$ In MLTT, List $(A)$ corresponds to the set of lists of elements of a set $A$. We will keep the intuition regarding the need to represent lists of objects but instead of using the inductive type $\operatorname{List}(A)$, we will use the inductive family of types $\operatorname{Vec}(A, n) . \operatorname{Vec}(A, n)$ and $\operatorname{List}(A)$ are very much alike, with the difference being mainly that $\operatorname{Vec}(A, n)$ involves an additional argument $n$ of type $N a t$, which

\footnotetext{
${ }^{20}$ Another interesting account of plurals is given by [31] using Girard's system F. It is shown that the basic properties of plurals can be effectively accounted for by using a second-order system like Girard's system F.
} 
counts the number of the elements in a list (that is why they are called vectors): ${ }^{21}$

(32) Vec: $($ A : Type)(n: Nat)Type

Now, collective predicates can be given types in a more appropriate way. For example, the collective predicate meet can be given the following type:

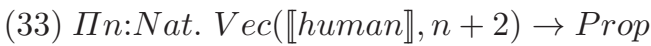

Please note that, as $n \geq 0$, an object of type $V e c(\llbracket h u m a n \rrbracket, n+2)$ has length of at least 2 or longer - this means that meet can only be applied to at least two people, but not less. Such more exact requirements are captured in typing by means of the inductive families like $\operatorname{Vec}(A, n)$.

Now, let us explain how to interpret sentences like (34):

(34) John and Mary met.

The above typing of meet assumes that the typing for humans can distinguish the number for the plural cases. In other words, this assumes that, collective and should be given the following type:

(35) $\Pi A: C N . \Pi n, m: N a t . V e c(A, n) \rightarrow \operatorname{Vec}(A, m) \rightarrow \operatorname{Vec}(A, n+m)$

Therefore, for example, assuming that $J: V e c(\llbracket h u m a n \rrbracket, 1)$ and $M: V e c(\llbracket h u m a n \rrbracket, 1)$, then $J$ and $M$ is of type $V e c(\llbracket h u m a n \rrbracket, 2)$. In order to type phrases like John and Mary, we need to introduce the following coercions, for every type $A$ :

$$
A<_{c} \operatorname{Vec}(A, 1)
$$

where the coercion $c$ maps any $a$ to $[a]$, the vector with only element $a$. With

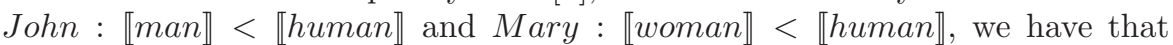

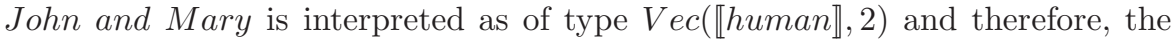
above sentence (34) gets interpreted as intended.

However, we are not done yet with collective predication, given that we have not yet discussed cases involving quantifiers. Such a case is shown below:

(36) Three men and five women met.

Given the type associated with quantifiers, the rule for collective coordination as this was given in (35) will not work. What we propose is to use a unit type which will encode both typings for collective and. There is no space here to

\footnotetext{
${ }^{21}$ See Chapter 9 of [15] for the formal definition of $\operatorname{Vec}(A, n)$. We omit the formal details here. Furthermore, and as suggested by an anonymous reviewer, one might consider using finite types (see for example Appendix B of [19]) instead of vector type. This seems to be a good suggestion and will be considered for future refinements of the proposals found in this paper.
} 
explain the notion of a unit type but let us say that such a type will encode both the typings in (37) via the coercions in $(38):{ }^{22}$

(37) and $_{1} \rrbracket: \Pi A: C N . \Pi n, m: N a t . V e c(A, n) \rightarrow \operatorname{Vec}(A, m) \rightarrow \operatorname{Vec}(A, n+m)$ $\llbracket$ and $_{2} \rrbracket: \Pi A:$ CN. $((\operatorname{Vec}(A, n) \rightarrow \operatorname{Prop}) \rightarrow \operatorname{Prop}) \rightarrow((\operatorname{Vec}(A, m) \rightarrow$ Prop $) \rightarrow$ Prop $) \rightarrow((\operatorname{Vec}(A, n+m) \rightarrow$ Prop $) \rightarrow$ Prop $)$

$(38) c_{1}($ and $)=\llbracket$ and $d_{1} \rrbracket$ and $c_{2}($ and $)=\llbracket a n d_{2} \rrbracket$

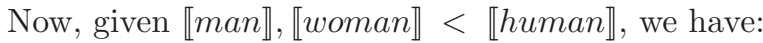

(39) and $(\llbracket$ three men $\rrbracket)(\llbracket$ five women $\rrbracket):((V e c(\llbracket$ human $\rrbracket, 3+5) \rightarrow$ Prop $) \rightarrow$ Prop)

Meet is applied to the above type and the sentence is well-typed.

Remark 1. Another way of dealing with collective predication is to assume a type for collective and that extends over the universe LType rather than $C N$. This rule will produce the following typing in case of quantifier coordination $V e c(((A \rightarrow$ Prop $) \rightarrow$ Prop $), n+m)$. However, given such a type since meet will not be able to apply assuming the type in (40). The solution in this case will be to have a unit type for meet, where one of the two types of the unit type is type lifted and turned into a functor taking a $G Q$ as an argument. We leave the discussion on the consequences and formalization of such proposal open due to space limitations.

One further welcoming extension of the account proposed is a straightforward explanation of the way the reciprocal each other functions in English. Verbs like meet are reciprocal predicates in the sense that they do not need an overt reciprocal to give rise to a reciprocal reading (basically what we have been calling the collective reading so far). For non-reciprocal predicates, there is the possibility of getting these readings via the use of each other. The idea is that each other in English turns a transitive predicate into an intransitive one whose sole argument is a vector $A: C N$ with $n$ of at least $2:^{23}$

(40) eeach $t$ ther $\rrbracket: \Pi A:$ CN. $(A \rightarrow A \rightarrow \operatorname{Prop}) \rightarrow(\operatorname{Vec}(A, n+2)$ Prop $)$

\footnotetext{
${ }^{22}$ Another possibility will be to assume that only the type relevant for the collective interpretation of GQs is needed. In this case, proper names can be interpreted collectively only in their GQ guise.

${ }^{23}$ If we want to generalize the rule to verbs with arity of more than two, we can use the following: eachother $\rrbracket: \Pi A: \mathrm{CN} .\left(A \rightarrow A \rightarrow A^{*} \rightarrow \operatorname{Prop}\right) \rightarrow(\operatorname{Vec}(A, 2) \rightarrow$ $A^{*}$ Prop), where $A^{*}$ stands for 0 or more $A$ arguments.
} 


\section{Interaction of Coordination and Copredication}

Dot-types have been successfully formalized in MTTs with coercive subtyping $[17,18]$, and an implementation of them in the proof assistant Plastic also exists [35]. We first summarize the account proposed for dot-types and then proceed and discuss the interaction between dot-types and coordination. We will use book as our prototypical example in presenting the account.

Book is assumed to be a dot-type having both a physical and an informational aspect. The type-theoretic formalization of this intuition proceeds as follows. Let PHY and INFO be the types of physical objects and informational objects, respectively. One may consider the dot-type $\mathrm{PHY} \bullet \mathrm{INFO}$ as the type of the objects with both physical and informational aspects. A dot-type is then a subtype of its constituent types: $\mathrm{PHY} \bullet \mathrm{INFO}<\mathrm{PHY}$ and $\mathrm{PHY} \bullet \mathrm{INFO}<\mathrm{INFO}$. A book may be considered as having both physical and informational aspects, reflected as:

$$
(*) \quad \llbracket b o o k \rrbracket<\mathrm{PHY} \bullet \mathrm{INFO} .
$$

Now, consider the following sentence:

(41) John picked up and mastered the book.

We assume the following typing for pick-up and master respectively:

$$
\begin{aligned}
& \llbracket p i c k \text { up } \rrbracket: \llbracket \text { human } \rightarrow \text { PHY } \rightarrow \text { Prop } \\
& \llbracket \text { master } \rrbracket: \llbracket \text { human } \rrbracket \rightarrow \mathrm{INFO} \rightarrow \text { Prop }
\end{aligned}
$$

Because of the above subtyping relationship $(*)$ (and contravariance of subtyping for the function types), we have

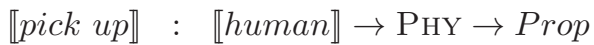

$$
\begin{aligned}
& <\llbracket \text { human } \rightarrow \text { PHY } \bullet \mathrm{INFO} \rightarrow \text { Prop } \\
& <\llbracket \text { human } \rightarrow \text { } \rightarrow \text { book } \rrbracket \rightarrow \text { Prop } \\
& \llbracket \text { master } \rrbracket: \llbracket \text { human } \rightarrow \text { INFO } \rightarrow \text { Prop } \\
& <\llbracket \text { human } \rightarrow \text { PHY } \bullet \mathrm{INFO} \rightarrow \text { Prop } \\
& <\llbracket \text { human } \rrbracket \rightarrow \llbracket \text { book } \rrbracket \rightarrow \text { Prop }
\end{aligned}
$$

Therefore, $\llbracket p i c k$ up and $\llbracket$ master $\rrbracket$ can both be used in a context where terms

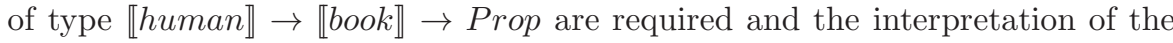
sentence (41) can proceed as intended.

The first case of interaction has already been introduced and involves examples like (41). It is to see how this is going to be predicted given what we have said. ${ }^{24}$

\footnotetext{
$\overline{{ }^{24} \text { See }[17,18]}$ for an account of this.
} 
The next step is to take a look at examples where two words with dot-types are coordinated. Such an example is shown below:

(42) The book and my lunch were sent by mistake to someone else.

In the above example we have two dot-types involved, PHY $\bullet$ INFO and PHY $\bullet$ EVENT, representing the types for book and lunch respectively. Let us see whether the rule for coordination we have along with the treatment of dot-types will give us the correct results.

We need to coordinate the two NPs:

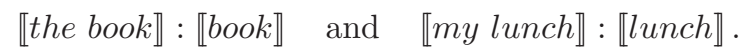

Furthermore, the passive send is of the following type:

$$
\llbracket \text { send }_{\text {pass }} \rrbracket: \text { HumAN } \rightarrow \text { PHY } \rightarrow \text { Prop } .
$$

Now, because

$$
\begin{gathered}
\llbracket b o o k \rrbracket<\mathrm{PHY} \bullet \mathrm{INFO}<\mathrm{PHY} \\
\llbracket l \text { lunch } \rrbracket<\mathrm{PHY} \bullet \mathrm{EVENT}<\mathrm{PHY}
\end{gathered}
$$

the above sentence (42) can be interpreted as intended. In other words, the coercive subtyping mechanism interacts with that for coordination correctly.

\section{Conclusions}

In this paper we presented an account of NL coordination using Type Theory with Coercive Subtyping. The issue of conjoinable types was taken care of by proposing an inductive type for coordination which extends over the universe of Linguistic Types, called LType. This type has been shown to be sufficient to explain the flexibility of NL coordination. We argued that a rule for NL coordination should in principle allow quantifier coordination and showed that the infelicitous quantifier combination cases are due to the inherent semantics of the quantifier combination under the coordinator in each case, along with general pragmatic implicatures associated with quantifiers (e.g. the quantity implicature for quantifiers some and most). Non-Boolean conjunction was accounted for, assuming that collective predicates take one vector argument representing plurality. A second rule for collective and was proposed which takes two vector arguments of $n$ and $m$ length and produces a vector type of length $n+m$. Lastly, the interaction of dot.types with coordination was briefly discussed. It was shown that the coordination account proposed in combination with the co-predication account as this was given in [18] gives the correct predictions.

\section{References}

1. The Agda proof assistant (version 2). Available from the web page: http://appserv.cs.chalmers.se/users/ulfn/wiki/agda.php (2008) 
2. Asher, N.: Lexical Meaning in Context: a Web of Words. Cambridge University Press (2012)

3. Bassac, C., M.B., Retor, C.: Towards a type-theoretical account of lexical semantics. Journal of Logic Language and Information 19, 229-245 (2010)

4. Boldini, P.: The reference of mass terms from a type-theoretical point of view. Paper from the 4th International Workshop on Computational Semantics (2001)

5. Callaghan, P., Luo, Z.: An implementation of LF with coercive subtyping and universes. Journal of Automated Reasoning 27(1), 3-27 (2001)

6. Church, A.: A formulation of the simple theory of types. J. Symbolic Logic 5(1) (1940)

7. The Coq Development Team: The Coq Proof Assistant Reference Manual (Version 8.3), INRIA (2010)

8. Curry, H., Feys, R.: Combinatory Logic, vol. 1. North Holland (1958)

9. Geach, P.: Reference and Generality: An examination of some Medieval and Modern Theories. Cornell University Press (1962)

10. Geurts, B.: Quantity Implicatures. Cambridge University Press (2010)

11. Hoeksema, J.: The semantics of non-boolean and. Journal of Semantics 6, 19-40 (1998)

12. Horn, L.: The border wars: a neo-gricean perspective. In: von Heusinger, K., Turner, K. (eds.) Where semantics meets pragmatics,s, pp. 21-46. Amsterdam: Elsevier. (2006)

13. Horn, L., R.: A Natural History of Negation. University of Chicago Press (1989)

14. Howard, W.A.: The formulae-as-types notion of construction. In: Hindley, J., Seldin, J. (eds.) To H. B. Curry: Essays on Combinatory Logic. Academic Press (1980)

15. Luo, Z.: Computation and Reasoning: A Type Theory for Computer Science. Oxford Univ Press (1994)

16. Luo, Z.: Coercive subtyping. Journal of Logic and Computation 9(1), 105-130 (1999)

17. Luo, Z.: Type-theoretical semantics with coercive subtyping. Semantics and Linguistic Theory 20 (SALT20), Vancouver (2010)

18. Luo, Z.: Contextual analysis of word meanings in type-theoretical semantics. In: Logical Aspects of Computational Linguistics (LACL'2011). LNAI 6736 (2011)

19. Luo, Z.: Common nouns as types. In: Bechet, D., Dikovsky, A. (eds.) Logical Aspects of Computational Linguistics (LACL'2012). LNCS 7351 (2012)

20. Luo, Z., Soloviev, S., Xue, T.: Coercive subtyping: theory and implementation. Submitted manuscript (2012)

21. Martin-Löf, P.: Intuitionistic Type Theory. Bibliopolis (1984)

22. Montague, R.: The proper treatment of quantification in ordinary English. In: Hintikka, J., Moravcsik, J., Suppes, P. (eds.) Approaches to Natural Languages (1973)

23. Montague, R.: Formal Philosophy. Yale University Press (1974)

24. Moortgat, M.: Categorial type logics. In: van Benthem, J., ter Meulen, A. (eds.) Handbook of Logic and Language. Elsevier/Mit press (1997)

25. Morril, G.: Type Logical Grammar: Categorial Logic of Signs. Kluwer Academic Publishers (1994)

26. Nordström, B., Petersson, K., Smith, J.: Programming in Martin-Löf's Type Theory: An Introduction. Oxford University Press (1990)

27. Partee, B., Rooth, M.: Generalized conjunction and type ambiguity. In: Bauerle, R., S.C., von Stechow, A. (eds.) Meaning, use, and interpretation of language. Mouton De Gruyter (1983) 
28. Pustejovsky, J.: The Generative Lexicon. MIT (1995)

29. Pustejovsky, J.: Meaning in Context: Mechanisms of Selection in Language. Cambridge Press (2005)

30. Ranta, A.: Type-Theoretical Grammar. Oxford University Press (1994)

31. Retoré, C.: Variable types for meaning assembly: a logical syntax for generic noun phrases introduced by 'most'. Recherches linguistiques de Vincennes 41, 83-102 (2012)

32. Sundholm, G.: Proof theory and meaning. In: Gabbay, D., Guenthner, F. (eds.) Handbook of Philosophical Logic III: Alternatives to Classical Logic. Reidel (1986)

33. Winter, Y.: Flexibility Principles in Boolean Semantics. MIT Press, New York (2002)

34. Winter, Y.: A unified semantic treatment of singular np coordination. Linguistics and Philosophy 19, 337-391

35. Xue, T., Luo, Z.: Dot-types and their implementation. Logical Aspects of Computational Linguistics (LACL 2012). LNCS 7351 (2012)

\section{A Implementations in Coq}

We use Coq's predifined Type Universe instead of LType. Bvector is needed for vectors (Require Import Bvector). The coercion $A<_{c} \operatorname{Vec}(A, 1)$ for proper nouns is not possible in Coq, so we have to introduce the coercions as separate entries.

\section{A.1 Conjoinable types}

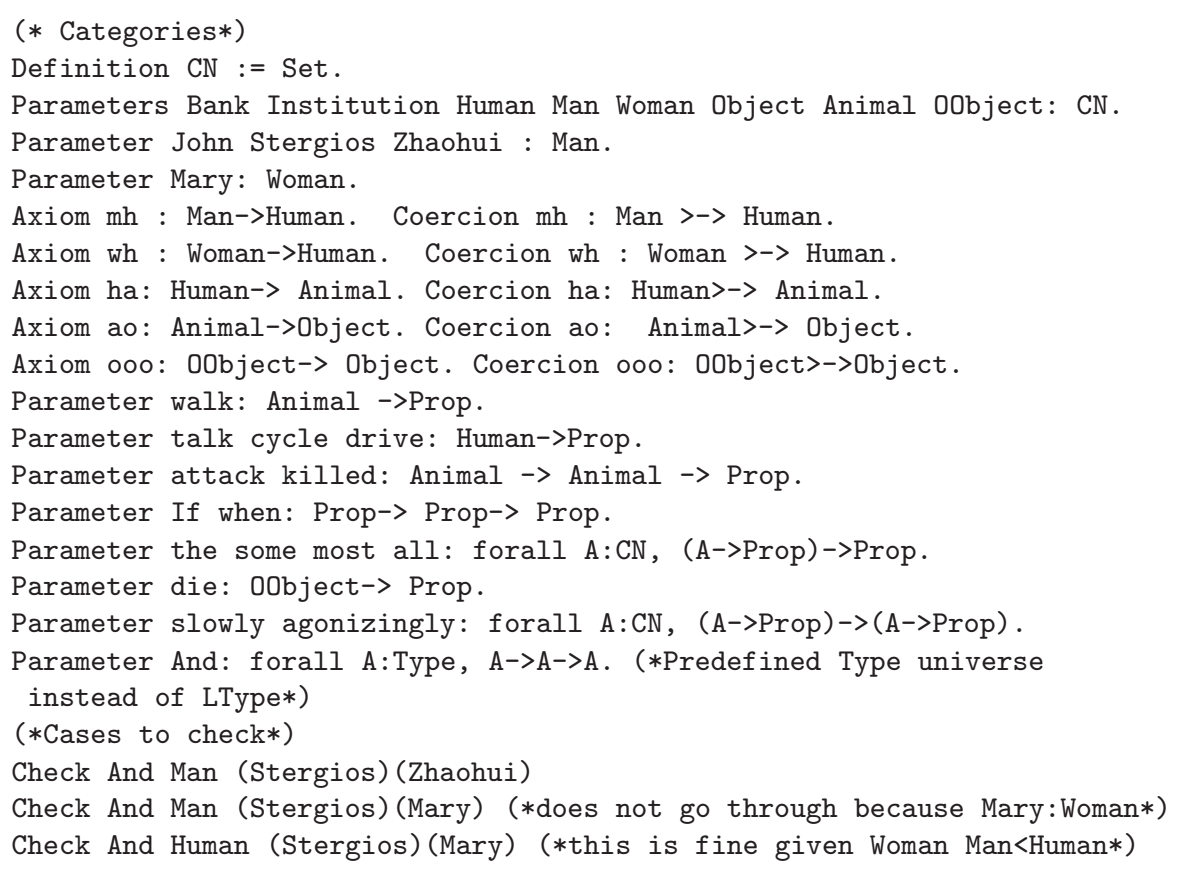


Check And ((Human->Prop)->Prop) (some Man) (some Woman) (*Quantifier NP coordination*)

Check And (forall A: CN, (A->Prop)->Prop) (some)(all). (*Quantifier coordination*)

Check And (Human->Prop) (cycle)(drive) ( $*$ VP coordination*)

Check And (forall A:CN, (A->Prop)->(A->Prop)) (slowly) (agonizingly). (*VP adverb coordination*)

Check And (Prop->Prop->Prop) (If)(when) (*subordinate conjunction coordination*)

\section{A.2 Non-Boolean Conjunction}

Require Import Bvector.

Variables $\mathrm{n}$ : nat.

Parameter meetc:forall $\mathrm{n}$ :nat, vector Human(n+2)->Prop. (*collective meet*).

Parameter John1 George1: vector Human 1.(*coercions do not work with vectors so we use Human instead of Man here*)

(*Unit type for collective And*)

Inductive OneAndc : Set := Andc.

Definition AndSem1 := forall A: CN,forall $\mathrm{n}$ :nat,forall $\mathrm{m}$ : nat, vector $(\mathrm{A})(\mathrm{n})$

$\rightarrow \operatorname{vector}(\mathrm{A})(\mathrm{m})->\operatorname{vector}(\mathrm{A})(\mathrm{n}+\mathrm{m})$.

Definition AndSem2 :=forall A: CN,forall $n$ :nat,forall m:nat, ((vector A n) $->$ Prop) $->$ Prop->

((vector A m) ->Prop)->Prop-> ( (vector A $(n+m))->$ Prop).

Parameter Andc1 : AndSem1.

Parameter Andc2 : AndSem2.

Definition a1 (a:OneAndc) : AndSem1 := Andc1. Coercion a1 : OneAndc >-> AndSem1.

Definition a2 (a:OneAndc) : AndSem2 := Andc2. Coercion a2 : OneAndc >-> AndSem2.

*Some interesting cases to check*

Check meetc 0 ((Andc:AndSem1 (Human)(1)(1)(John1)(George1)) (*John and George met, with both George and John of lower type*)

\section{A.3 Co-predication}

(* Phy dot Info *)

Parameter Phy Phy1 Info : CN.

(*Phy1 should be taken to be the same as Phy*)

Record PhyInfo: CN := mkPhyInfo $\{$ phy :> Phy; info :> Info $\}$.

Parameter Book: PhyInfo.

Parameter Event : CN

Record EventPhy : CN := mkEventPhy $\{$ event :> Event; phy1 :> Phy1\}. (*Phy1 is used because Phy

cannot be used twice*)

Parameter lunch: EventPhy.

Axiom po: Phy->Object. Coercion po:Phy>->Object.

Axiom pp: Phy1->Phy. Coercion pp: Phy1>->Phy. (*We introduce this coercion to mean that the two

Phy and Phy1 are the same.*)

Parameter was_given_to_someone_else: Object->Prop.

*Interesting case to check*)

Check was_given_to_someone_else (And(Object)(Book)(lunch)). 